\title{
Theoretical Analysis of Tuning and Sensitivity Improvement of Surface Plasmon Resonance Biosensor employing Heterostructures of Titanium Disilicide and Graphene
}

\section{Balaji V R ( $\sim$ balajivr@skcet.ac.in )}

Sri Krishna College of Engineering and Technology

\section{Research Article}

Keywords: Titanium disilicide nanosheet, Graphene, surface plasmon resonance, biosensor, sensitivity enhancement.

Posted Date: August 9th, 2021

DOl: https://doi.org/10.21203/rs.3.rs-705251/v1

License: (c) (1) This work is licensed under a Creative Commons Attribution 4.0 International License. Read Full License 


\title{
Theoretical Analysis of Tuning and Sensitivity Improvement of Surface Plasmon Resonance Biosensor employing Heterostructures of Titanium Disilicide and Graphene
}

\author{
V. R. Balaji* \\ Department of ECE, Sri Krishna College of Engineering and Technology, Coimbatore, India. \\ Email: *balajivr@skcet.ac.in
}

\begin{abstract}
In this work, a surface plasmons resonance-based biosensor is theoretically and mathematically investigated. The proposed sensor is a multilayered structure of the titanium disilicide and graphene along with Ag metal. These layers are stacked over the BK7 prism. The metal, titanium disilicide, and graphene layer thickness are taken as $45 \mathrm{~nm}, 2 \mathrm{~nm}$, and $1.7 \mathrm{~nm}$, respectively. The titanium disilicide is an air-stable 2-D material. The performance of the biosensor is analyzed using the attenuated total reflection (ATR) method. The proposed configuration has a maximum sensitivity of $183.4^{\circ} / \mathrm{RIU}, 47.45 \%$ higher than the conventional sensor. The designed sensor has wide applications in chemical, medical, and biological analyte sensing.
\end{abstract}

Keywords: Titanium disilicide nanosheet, Graphene, surface plasmon resonance, biosensor, sensitivity enhancement.

\section{Introduction}

Surface plasmons resonance (SPR) is the emerging technology for analyzing the drastic change in the sensing medium's refractive index when the analyte contacts the sensor [1]-[3]. SPR is the oscillations of the electrons in the conduction band, which are stimulated due to the interaction of the optical signal at the interface of the metal (negative permeability) and dielectric (positive permeability) material. SPRs are sensible for any change at the boundary because the surface plasmons wave is on the boundary [4]. SPR sensors are in the trends globally due to a variety of applications in the different areas like biosensing, monitoring of the environment, medical field, food and safety, gas detection, temperature analysis, pressure measurement, concentration analysis of the fluid, analyze different components of the blood, and as well as real-time observation of the molecular interaction of the molecules in the microbiology and biochemistry with ultra-high sensitivity, and level free detection. It has applications in optical fields like optical modulators and plasma detectors [5]-[8]. Various sensors using SPR technology have been proposed, but researchers, academicians, and scientists are putting their efforts into improving the sensor's 
performance [9], [10]. The SPR sensors are implemented using either angle interrogation or wavelength interrogation method to detect the sensing layer refractive index variation when the analyte comes in contact with it. In the angle interrogation technique, the Kretschmann configuration is used for the detection of the analyte. In this configuration, a monochromatic light source generates the surface plasmons polaritons at the metal and dielectric boundary [11]. It is responsible for propagating and generating the surface plasmons wave (SPW). However, in the wavelength interrogation method, a polychromatic light beam is required to stimulate SPPs [12], [13].

The SPR sensor can predict earlier diseases during the diagnostic, and preventive action can be taken. Due to these advantages of the SPR sensors, researchers are putting their efforts into designing suitable sensors with high sensitivity. Various types of sensors have been proposed, such as classical optical fiber and photonic crystal sensor, grating-based, and prism-coupled-based sensors [14]-[17]. In addition, prism coupling-based sensors using Kretschmann geometry have been investigated, and attempts have been made to improve performance parameters. However, the nanostructured sensors' performance has been enhanced in the past few years due to the advancement in the nanostructure's fabrication technology [18]. Hence, the Kretschmann structure-based sensor is a promising method for sensing purposes. The sensitivity, detection accuracy, full width half maximum (FWHM), quality factor, etc., have been improvised using the modified multilayer Kretschmann structure [19]-[21].

The metal layer of silver (Ag) is regularly used in the conventional biosensor; sometimes, gold $\mathrm{Au})$ is preferred [5]. This material shows a sharp dip in the resonance curve near the infrared region and near-infrared region, i.e., it reflects that the sensor produces high sensitivity due to sharp dip. Au is a preferred metal for the sensor because it gives high resonance angle change due to variation in the refractive index of the sensing medium, causes high sensitivity. Furthermore, $\mathrm{Au}$ is stable material in the air and shows chemical internees as well. But Ag metal layer base sensor gives better accuracy than gold, also indicates a shaper dip on the resonance curve with higher contrast, and FWHM is lower than the Au. But Ag shows low stability because it is easily oxidized when it comes in contact with sensing material. Due to the oxidation of the Ag layer, the sensitivity of the sensor decreases. A bimolecular recognizing element (BRE) is used to mitigate this oxidation problem and enhance the sensor's performance [22]. 
Experimentally, it comes to know that 2-D materials such as black phosphorus and graphene as BREs can improve the sensitivity and performance parameters of the SPR sensors. Graphene is the slimmest artificial planner material, composed of carbon atoms organized hexagonally in a plane. Graphene has extraordinary optical, electrical, and mechanical properties. It has commendable tenability, low loss, high captivity, large surface-to-volume ratio, enhances the ability to contact the analyte's molecule properly, and facilitates detecting aromatic compounds due to its $\pi$-stacking structure [23], [24]. When a layer of graphene is stacked over the thin film of the metal or attached over the metallic nanoparticle such as Ag, a strong bonding may be stimulated at the boundary of the metallic/graphene due to charge transfer at the interface. This is the reason why a strong electric field is enhanced at the metal-dielectric interface. Black phosphorus (BP) is also BRE material, but it is unstable in air and water [25], [26]. Therefore, BP is the best suitable material for gas sensing. Graphene is the best choice for designing the biosensor and shows the best stability in contact with air or aqueous solution. Many researchers have shown their interest in developing hybrid multilayered biosensors by utilizing the layers of Ag, graphene, and some other 2-D materials. Rahman et al. theoretically investigated a biosensor using BK7 prism, $\mathrm{PtSe}_{2}$, 2D materials, and maximum sensitivity 194\%/RIU is achieved [5].

The proposed sensor is designed for medical diagnostics and biomedical detection applications. Xiang et al. suggest an SPR based gas detection sensor using germanium prism and graphene sheet, and maximum sensitivity $147^{\circ} / \mathrm{RIU}$ is obtained [19]. Pal and Jha theoretically analyze an SPR biosensor. Investigated configuration use BK7 prism, the bimetallic layer of Ag, barium titanate, and graphene nanosheet [27]. Rouf and Haque proposed an ultra-sensitive SPR sensor consisting of a bimetallic $\mathrm{Ag}$ and Au layer and titanium and indium phosphide. The authors have suggested that the proposed sensor can detect variation of 1/1000 of RIU of the sensing medium [8]. The $\mathrm{TiSi}_{2}$ is a transition metal disilicide material having an orthorhombic heterostructure structure [28]. It is a very stable material in the air, but the oxide formation occurs when it comes in contact with an aqueous solution [29]. To prevent oxide formation protective layer of graphene is used. The bulk form of $\mathrm{TiSi}_{2}$ provides a direct bandgap of $0.5 \mathrm{eV}$ at room temperature and shows high absorption efficiency [30]. On the other hand, titanium disilicide has low resistivity properties best to design biosensors. 
In this work, a hybrid multilayer sensor based on Ag metal, titanium disilicide, and graphene has been designed to analyze the conventional structure comprehensively. The sensor is analyzed using an angular interrogation approach based on attenuated total reflection (ATR). The manuscript is structured in the following manner: Section 2 consists of design consideration and theoretical cum mathematical modeling of the proposed sensor. Section 3 explains results and discussion, while the conclusion of the work is provided in section 4.

\section{Design and modeling of the biosensor}

Figure 1 depicts our design of the SPR biosensor in a typical Kretschmann configuration. The TM polarized optical signal of $633 \mathrm{~nm}$ is incident at one surface of the BK7 prism, and the refracted optical signal is received at the other end.

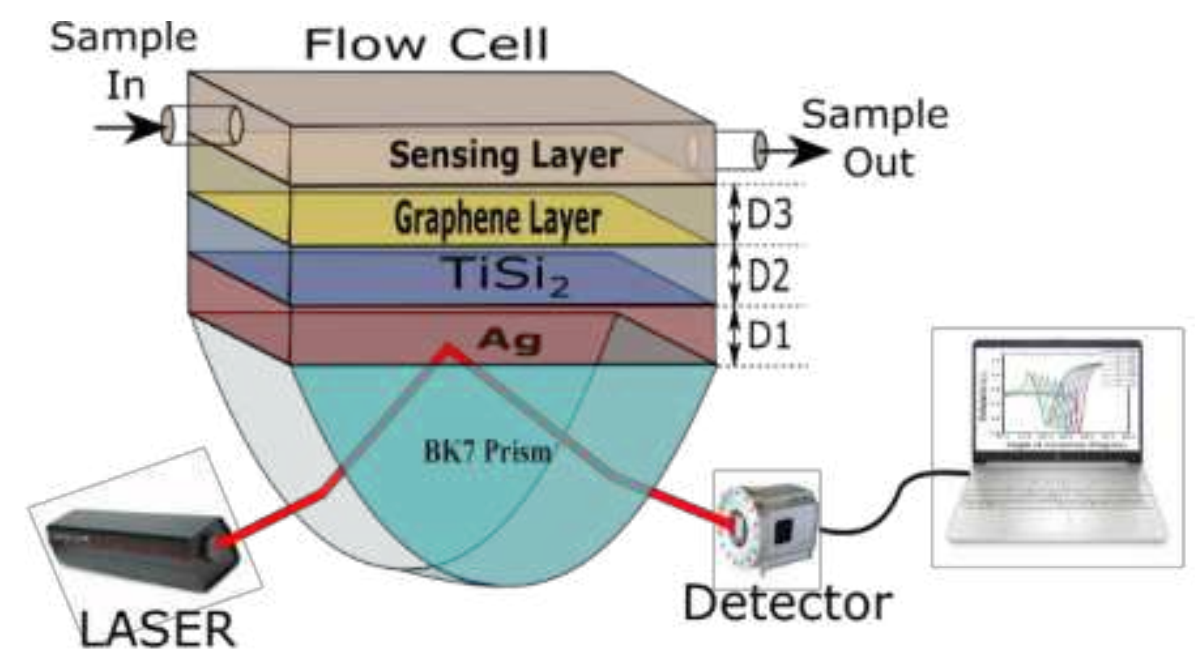

Figure 1: Layered structure of biosensor based on $\mathrm{Ag}, \mathrm{TiSi}_{2}$, and graphene.

The prism is coated with a thin layer of $\mathrm{Ag}$ (a single layer of thickness $D_{1}=45 \mathrm{~nm}$ ), which in turn is covered by a 2-D titanium disilicide (thickness $D_{2}=M * 2 \mathrm{~nm}$ where $M$ is an integer with values $0,1,2$, etc. $M$ represents the number of TiSi2 layers), followed by graphene layers (thickness $D_{3}=$ $G * 0.34 \mathrm{~nm}$, where $G$ is an integer with values $0,1,2$, etc. $G$ represents the number of graphene layers). The graphene layer is sandwiched between $\mathrm{TiSi}_{2}$ film and the sensing layer. The graphene layer improves the macromolecular captivation of the sensor. The BK7 prism is used as a coupling prism, the RI of the prism is given as [21]:

$n_{p B K 7}=\sqrt{1+\frac{1.03961212 \lambda^{2}}{\lambda^{2}-0.00600069867}+\frac{0.231792344 \lambda^{2}}{\lambda^{2}-0.0200179144}+\frac{1.01046945 \lambda^{2}}{\lambda^{2}-103.560653}}$ 
where $\lambda$ is the wavelength of the applied optical signal. The RI of the metal layer (Ag) is expressed using the Drude model, $n_{A g}=\sqrt{1-\left(\lambda^{2} \lambda_{c}\right) /\left[\lambda_{p}^{2}\left(\lambda_{c}+i \lambda\right)\right]}$, where $\lambda_{c}=17.614 \mu \mathrm{m}$ and $\lambda_{p}=$ $0.14541 \mu \mathrm{m}$. The RI $\left(n_{f}\right)$ of titanium disilicide at $2 \mathrm{~nm}$ thickness is $2.70008+i * 2.939449$ at $633 \mathrm{~nm}$ [31]. The RI of graphene is given as $n_{G}=3.0+i C_{1} * \lambda / 3$ with $C_{1} \approx 5.446 \mu \mathrm{m}^{-1}, \lambda$ is the wavelength of the applied optical signal [32]. The RI of the sensing layer is taken $n_{s}=1.33$. Figure 2 shows the real and imaginary part of the refractive index of TiSi2 for visible range.

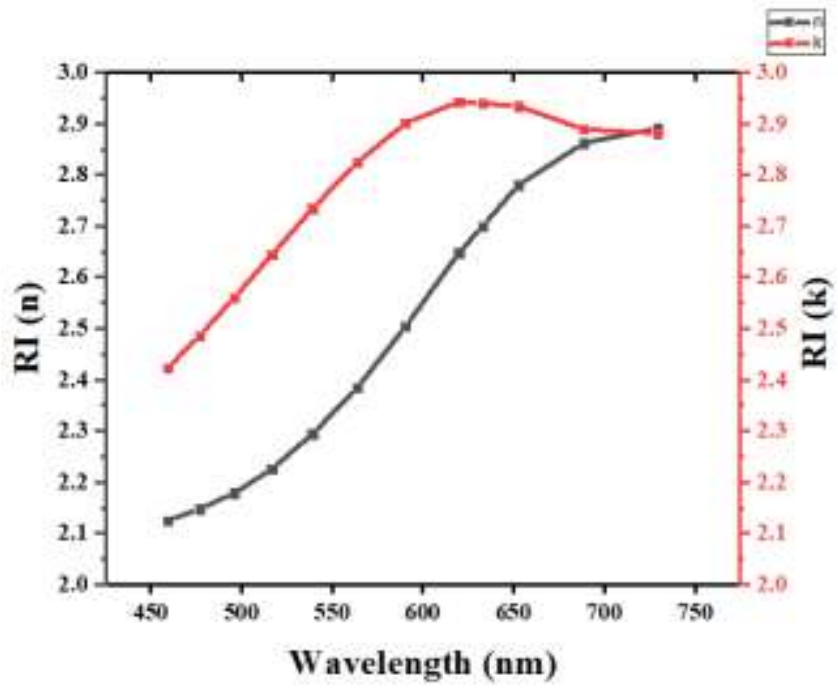

Figure 2: Refractive index of titanium disilicide ( $\mathrm{n}$ is the real part and $\mathrm{k}$ is the imaginary part) for the wavelength from $455 \mathrm{~nm}$ to $730 \mathrm{~nm}$

Detailed numerical analysis by varying thickness of metal layers was carried out and simulated to get minimum reflectance for the geometry to find optimal thicknesses (for maximum sensitivity) of Ag metal coatings. The sensing performance of the systems is estimated using the Fresnel equations. Finally, the reflectance is calculated by the formula:

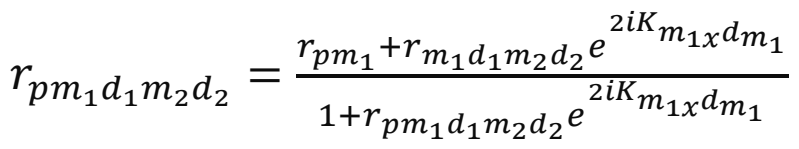

where

$$
\begin{aligned}
& K_{i x}=\left(\left(\frac{2 \pi}{\lambda}\right)^{2} \varepsilon_{i}-K_{z}^{2}\right)^{1 / 2} ; i=p, m_{1} d_{1} d_{2} \\
& r_{p m}=\frac{\left(\varepsilon_{m} K_{p x}-\varepsilon_{p} K_{m x}\right)}{\left(\varepsilon_{m} K_{p x}-\varepsilon_{p} K_{m x}\right)} ; r_{m d}=\frac{\left(\varepsilon_{d} K_{m x}-\varepsilon_{d} K_{d x}\right)}{\left(\varepsilon_{d} K_{m x}-\varepsilon_{m} K_{d x}\right)}
\end{aligned}
$$

In the subscribes, prism is denoted by $p$, metal by $m$, and dielectric by $d$, respectively. 
The dip in the resonance curve can be written as:

$$
K_{s p}=\frac{2 \pi}{\lambda} n_{0} \operatorname{Sin} \theta_{\mathrm{SPR}}=\operatorname{real}\left(\frac{2 \pi}{\lambda} \sqrt{\frac{\varepsilon_{m} \varepsilon_{s}}{\varepsilon_{m}+\varepsilon_{s}}}\right)
$$

$n_{0}$ is the RI of the coupling prism, $\varepsilon_{s}$ and $\varepsilon_{m}$ are dielectric constants of the sample and metal, respectively, angle of resonance is $\theta_{\mathrm{SPR}}$, and $K_{s p}$ is the propagation constant. The reflectance is defined as $R_{P}=\left|r_{p m_{1} d_{1} d_{2}}\right|^{2}$, where $r_{p m_{1} d_{1} d_{2}}$ denotes the reflection coefficient of the incident optical signal. Sensitivity is represented as $S_{n}=\delta \theta_{S P R} / \delta n_{s}$, where $\delta \theta_{S P R}$ and $\delta n_{s}$ represent the change in the resonance angle and the change in RI of the sensing layer, respectively. The consolidated design parameters for the implemented biosensors are listed in Table 1.

Table 1. Designing parameters for the proposed biosensor

\begin{tabular}{|c|c|c|c|c|}
\hline \multirow{2}{*}{$\begin{array}{c}\text { Film of the } \\
\text { materials }\end{array}$} & \multirow{2}{*}{ Used material } & \multicolumn{2}{|c|}{ RI of the material at $633 \mathrm{~nm}$} & Thickness (nm) \\
\cline { 3 - 5 } & & Real part (n) & Imaginary part (k) & \\
\hline Layer I & Prism BK7 & 1.5151 & - & - \\
\hline Layer II & Ag (Metal) & 0.056253 & 4.2760 & 45 \\
\hline Layer III & Titanium Disilicide & 2.70008 & 2.939449 & 2 \\
\hline Layer IV & Graphene & 3.0 & $C_{1} * \lambda / 3$ & 0.34 \\
\hline
\end{tabular}

\section{Result and discussion}

The conventional SPR sensor (Prism/Metal/Analyte) has limited sensitivity with the range of analyte refractive index. Hence, better performance can be achieved with some modification in the conventional SPR sensor structure. These changes include multilayer structure or 2D materials like graphene, black phosphorus, platinum diselenide, barium titanate, etc. has been used in SPR sensor for the performance improvement. Here, $\mathrm{TiSi}_{2}$ and the $2 \mathrm{D}$ material graphene have been used to increase the sensitivity of the proposed SPR sensor. This SPR sensor can be used for an extensive dynamic range of analyte RI from 1.332 to 1.382 RIU. Three-layered configurations are considered, as shown in Fig. 1. For better understanding, all three configurations of Fig. 1 are considered case-I, case-II, and case-III. The minimum value of the reflectance of $0.18,0.198$, 0.208 and SPR angles $68^{\circ}, 74.8^{\circ}, 76^{\circ}$ are calculated for case-I, case-II, and case-III, respectively depicted in Fig. 2. It is observed from the analysis of Fig. 2 that as we increased the number of the layer in conventional SPR structure contains prism and metal layer, the $R_{\min }$ is increased gradually, and $\theta_{S P R}$ is increased monotonically. This indicates that the case-II and case-III 
sensitivity are higher than the case-I, as shown in Fig. 3. The sensitivity for the analyte RI of 1.332 to 1.382 RIU for the conventional SPR sensor varies from 115.4 to 154.8 Degree/RIU, 122.6 to 169.2 Degree/RIU with the single layer of $\mathrm{TiSi}_{2}$, and 124.8 to 173 Degree/RIU with the singlelayer $\mathrm{TiSi}_{2}$ and single layer of graphene. Table 2 shows the sensor's sensitivity for the conventional sensor (Ag layer only), $\mathrm{Ag} / \mathrm{TiSi}_{2}$, and $\mathrm{Ag} / \mathrm{TiSi}_{2} /$ graphene layered sensor.

Table 2: Sensitivity of the sensor at different layers

\begin{tabular}{|c|c|c|c|}
\hline Structure $(\mathrm{BK} 7+)$ & $\theta_{S P R}$ & $\Delta \theta_{S P R}$ & Sensitivity (Degree/RIU) \\
\hline $\mathrm{Ag} /$ Analyte & 73.585 & 0.1783 & 154.8 \\
\hline $\mathrm{Ag} / \mathrm{TiSi}$ /Analyte & 75.087 & 0.846 & 169.2 \\
\hline $\mathrm{Ag} / \mathrm{TiSi}{ }_{2} /$ Graphene/Analyte & 75.503 & 0.865 & 173 \\
\hline
\end{tabular}

The further enhancement of the sensitivity is done with an increment of the number of the graphene layer. The graphene layer number varies from 1 to 6 and plots the reflection spectrum as shown in Fig. 4 (a). The minimum reflectance of the SPR curve increased with an increase in the graphene layer due to the interaction of the SPP and incident light gets violated. Also, the resonance angle shift is increased because the sensitivity also increased for the increased graphene layer. Figure 4 (b) depicts the maximum sensitivity achieved for changing the graphene layer from 1 to 6 is 173 Degree/RIU, 176.4 Degree/RIU, 179.8 Degree/RIU 182 Degree/RIU, 183.4 Degree/RIU, and 183.2 Degree/RIU, respectively.

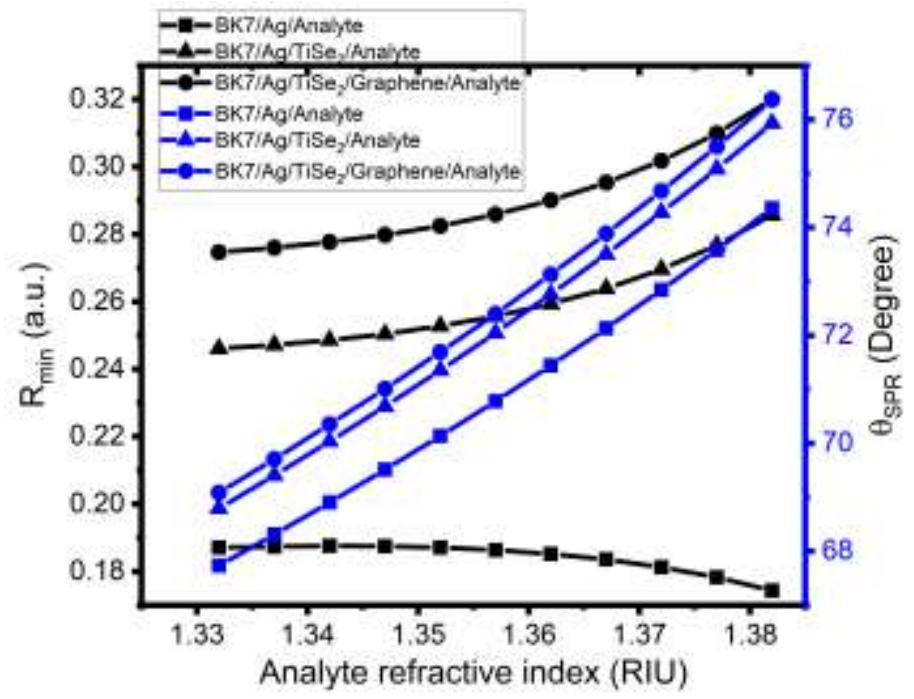

Fig. 2: relation between $R_{\min }$ and $\theta_{S P R}$ w.r.t analyte refractive index with $\mathrm{Ag} / \mathrm{TiSi}_{2} /$ graphene layers 


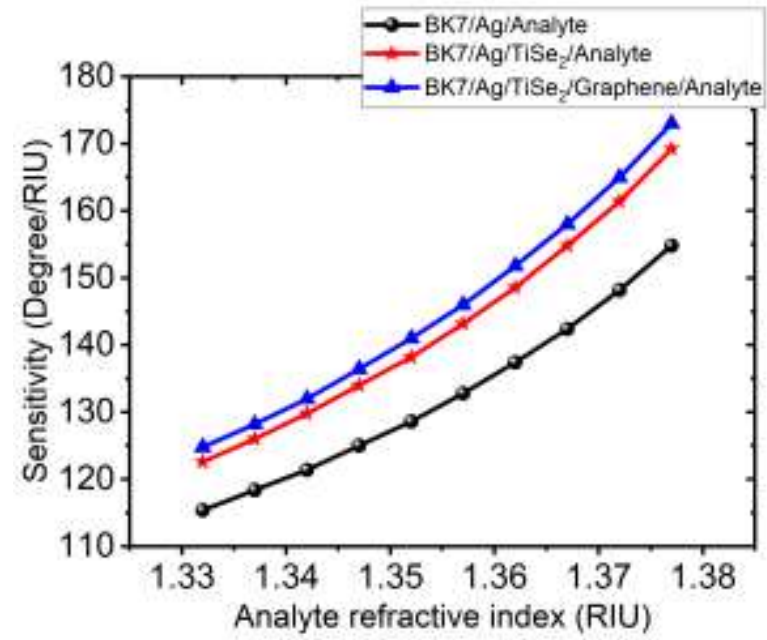

Fig. 3: plot of sensitivity vs. refractive index of the analyte.

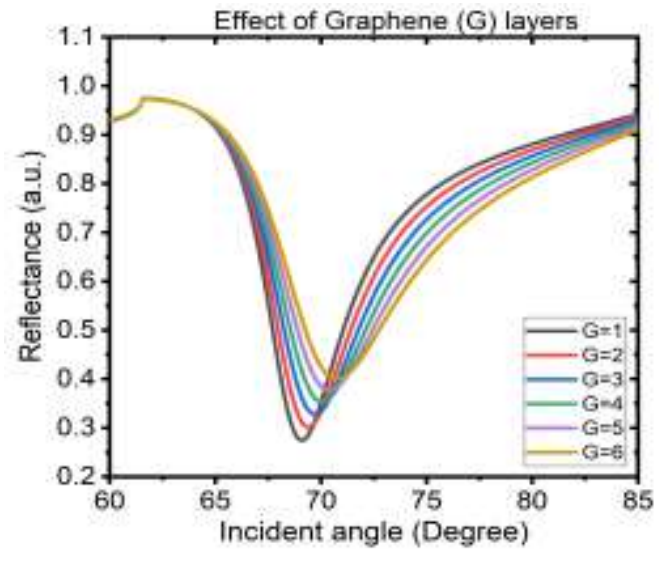

(a)

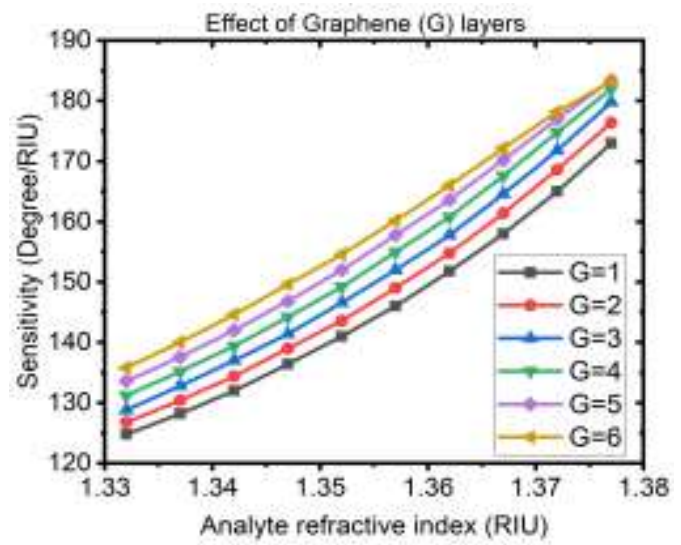

(b)

Fig. 4 (a): variation in reflectance w.r.t incident angle for different graphene layers (b): plot of sensitivity vs. refractive index for different graphene layers.

The proposed SPR sensor's sensitivity enhancement compared with the previously published work and is shown in Table 3. The proposed sensor has the sensitivity enhancement of $18.48 \%$ and $8.39 \%$ from the conventional SPR sensor and conventional SPR sensor with the only $\mathrm{TiSi}_{2}$.

Table 3: Comparison of the proposed work with previously reported work

\begin{tabular}{|c|c|c|c|}
\hline Configuration With BK7 Prism & Wavelength (nm) & Sensitivity & Ref. \\
\hline Ag, franckeite & 633 & $188^{\circ} / \mathrm{RIU}$ & [4] \\
\hline $\mathrm{Ag}, \alpha-\mathrm{SnSe}$ & 633 & $178^{\circ} / \mathrm{RIU}$ & [33] \\
\hline $\mathrm{Ag} / \mathrm{Au} . \mathrm{PtSe}_{2}$ & 633 & $162 / 165^{\circ} / \mathrm{RIU}$ & [5] \\
\hline $\mathrm{Ag} / \mathrm{TiSi}_{2} / \mathrm{Graphene}$ & 633 & $183.4^{\circ} / \mathrm{RIU}$ & Proposed work \\
\hline
\end{tabular}




\section{Conclusion}

A hybrid SPR biosensor based on the orthorhombic heterostructure of $\mathrm{TiSi}_{2}$ with 2-D materials is proposed for sensitivity enhancement. Ag, $\mathrm{TiSi}_{2}$, and graphene layers with thicknesses of $45 \mathrm{~nm}$, $2 \mathrm{~nm}$, and $1.7 \mathrm{~nm}$, respectively, are used in the design. The biosensor's sensitivity is also affected by the thickness of the graphene layers. A single layer of $\mathrm{TiSi}_{2}$ and a five-graphene layer yielded a maximum sensitivity of 183.4 Degree/RIU for the biosensor. It should be highlighted that the proposed systems have a greater sensitivity of 47.4 times that of traditional Ag-based sensors. With such strong sensing capabilities, the suggested sensor could be a good choice for detecting various liquid analytes in biosensing applications.

Code Availability: Not applicable.

Funding Information: No funding available.

Conflicts of interest/Competing interests: The author declare that he has no conflict of interest. Availability of data and material: No data available.

\section{Authors' contributions}

VRB Formulated the problem statement wherein giving the theoretical background for SPR biosensor. He has designed the complete manuscript.

\section{Ethics approval}

Not applicable. The work presented in this manuscript is mathematical modelling only for the proposed biosensor. No experiment was performed on the human body and/or living organism/ animal. So, an ethical approval from an ethical committee is not required.

\section{Consent to participate}

I am willing to participate in the work presented in this manuscript.

\section{Consent for publication}

The author has given their consent to publish this work.

\section{References}

[1] J. A. Schuller, E. S. Barnard, W. Cai, Y. C. Jun, J. S. White, and M. L. Brongersma, "Plasmonics for extreme light concentration and manipulation," Nat. Mater., vol. 9, no. 3, pp. 193-204, 2010.

[2] D. K. Gramotnev and S. I. Bozhevolnyi, "Plasmonics beyond the diffraction limit," Nat. Photonics, vol. 4, no. 2, pp. 83-91, 2010. 
[3] V. E. Bochenkov, M. Frederiksen, and D. S. Sutherland, "Enhanced refractive index sensitivity of elevated short-range ordered nanohole arrays in optically thin plasmonic Au films," Opt. Express, vol. 21, no. 12, p. 14763, 2013.

[4] S. Gan, Y. Zhao, X. Dai, and Y. Xiang, "Sensitivity enhancement of surface plasmon resonance sensors with 2D franckeite nanosheets," Results Phys., vol. 13, p. 102320, 2019.

[5] M. M. Rahman, M. M. Rana, M. S. Rahman, M. S. Anower, M. A. Mollah, and A. K. Paul, "Sensitivity enhancement of SPR biosensors employing heterostructure of PtSe2 and 2D materials," Opt. Mater. (Amst)., vol. 107, p. 110123, 2020.

[6] H. Fu, S. Zhang, H. Chen, and J. Weng, "Graphene enhances the sensitivity of fiber-optic surface plasmon resonance biosensor," IEEE Sens. J., vol. 15, no. 10, pp. 5478-5482, 2015.

[7] Y. Xiang, J. Zhu, L. Wu, Q. You, B. Ruan, and X. Dai, "Highly Sensitive Terahertz Gas Sensor Based on Surface Plasmon Resonance with Graphene," IEEE Photonics J., vol. 10, no. 1, pp. 1-7, 2018.

[8] H. K. Rouf and T. Haque, "Performance enhancement of Ag-Au bimetallic surface plasmon resonance biosensor using InP," Prog. Electromagn. Res. M, vol. 76, pp. 31-42, 2018.

[9] A. Nisha, P. Maheswari, P. M. Anbarasan, K. B. Rajesh, and Z. Jaroszewicz, "Sensitivity enhancement of surface plasmon resonance sensor with 2D material covered noble and magnetic material (Ni)," Opt. Quantum Electron., vol. 51, no. 19, 2019.

[10] M. H. H. Hasib, J. N. Nur, C. Rizal, and K. N. Shushama, "Improved Transition Metal Dichalcogenides-Based Surface Plasmon Resonance Biosensors," Condens. Matter, vol. 4, no. 2, p. 49, 2019.

[11] N. Mudgal and P. Y. J. A. G. SIngh, "BaTiO 3 -Graphene-Affinity Layer - Based Surface Plasmon Resonance ( SPR ) Biosensor for Pseudomonas Bacterial Detection," Plasmonics, vol. 15, pp. 12211229, 2020.

[12] J. Li, D. Han, J. Zeng, J. Deng, N. Hu, and J. Yang, "Multi-channel surface plasmon resonance biosensor using prism-based wavelength interrogation," Opt. Express, vol. 28, no. 9, p. 14007, 2020.

[13] B. Karki, S. Sharma, A. Pal, and Y. Singh, "Sensitivity Enhancement of Surface Plasmon Resonance Biosensor with 2-D Franckeite Nanosheets," Plasmonics, 2021.

[14] S. Firdous, S. Anwar, and R. Rafya, "Development of surface plasmon resonance (SPR) biosensors for use in the diagnostics of malignant and infectious diseases," Laser Phys. Lett., vol. 15, p. 065602, 2018.

[15] S. Dinodiya, B. Suthar, and A. Bhargava, "Photonic crystal sensors: Physics and applications," in AIP Conference Proceedings, 2018, vol. 1953, p. 060016.

[16] S. K. Chamoli, S. C. Singh, and C. Guo, "Design of Extremely Sensitive Refractive Index Sensors in Infrared for Blood Glucose Detection," IEEE Sens. J., vol. 20, no. 9, pp. 4628-4634, 2020.

[17] S. K. Chamoli, S. C. Singh, and C. Guo, "1-D Metal-Dielectric-Metal Grating Structure as an UltraNarrowband Perfect Plasmonic Absorber in the Visible and Its Application in Glucose Detection," Plasmonics, vol. 15, no. 5, pp. 1339-1350, 2020.

[18] L. Kong, J. Lv, Q. Gu, Y. Ying, X. Jiang, and G. Si, "Sensitivity-enhanced spr sensor based on graphene and subwavelength silver gratings," Nanomaterials, vol. 10, no. 11, pp. 1-12, 2020.

[19] T. Srivastava and R. Jha, "Black Phosphorus: A New Platform for Gaseous Sensing Based on Surface Plasmon Resonance," IEEE Photonics Technol. Lett., vol. 30, no. 4, pp. 319-322, 2018.

[20] O. Tabasi and C. Falamaki, "Recent advancements in the methodologies applied for the sensitivity enhancement of surface plasmon resonance sensors," Anal. Methods, vol. 10, no. 32, pp. 3906-3925, 2018.

[21] Y. Singh and S. K. Raghuwanshi, "Electromagnetic wave sensors Sensitivity Enhancement of the Surface Plasmon Resonance Gas Sensor With Black Phosphorus," IEEE Sensors Lett., vol. 3, no. 12, pp. 1-4, 2019.

[22] M. Saifur Rahman, M. S. Anower, L. Bin Bashar, and K. A. Rikta, "Sensitivity analysis of graphene coated surface plasmon resonance biosensors for biosensing applications," Sens. Bio-Sensing Res., vol. 16, no. October, pp. 41-45, 2017.

[23] B. A. Prabowo et al., "Graphene-based Portable SPR Sensor for the Detection of Mycobacterium 
tuberculosis DNA Strain," Procedia Eng., vol. 168, pp. 541-545, 2016.

[24] U. S. P. R. Biosensor, P. K. Maharana, P. Padhy, and R. Jha, "On the Field Enhancement and Performance of an," vol. 25, no. 22, pp. 2156-2159, 2013.

[25] A. Srivastava and Y. K. Prajapati, "Effect of sulfosalt and polymers on performance parameter of SPR biosensor," Opt. Quantum Electron., vol. 52, no. 10, pp. 1-14, 2020.

[26] B. Meshginqalam and J. Barvestani, "Performance Enhancement of SPR Biosensor Based on Phosphorene and Transition Metal Dichalcogenides for Sensing DNA Hybridization," IEEE Sens. J., vol. 18, no. 18, pp. 7537-7543, 2018.

[27] A. Pal and A. Jha, "A theoretical analysis on sensitivity improvement of an SPR refractive index sensor with graphene and barium titanate nanosheets," Optik (Stuttg)., vol. 231, p. 166378, 2021.

[28] A. Borghesi, A. Piaggi, G. Guizzetti, F. Levy, M. Tanaka, and H. Fukutani, "Optical properties of single-crystal titanium disilicide," Phys. Rev. B, vol. 40, no. 3, pp. 48-55, 1989.

[29] Y. Lin, S. Zhou, X. Liu, S. Sheehan, and D. Wang, "TiO 2/TiSi 2 heterostructures for highefficiencyphotoelectrochemical H 2 O splitting," J. Am. Chem. Soc., vol. 131, no. 8, pp. 2772-2773, 2009.

[30] A. A. Kovalevskii, V. A. Labunov, A. S. Strogova, and V. V. Tsybul'skii, "Formation of semiconductor titanium disilicide," Tech. Phys., vol. 61, no. 9, pp. 1343-1345, 2016.

[31] Filmetrics, "URL of RI of TiSi2," KLA Corporation. .

[32] X. Zhao et al., "Sensitivity enhancement in surface plasmon resonance biochemical sensor based on transition metal dichalcogenides/graphene heterostructure," Sensors, vol. 18, no. 7, p. 2056, 2018.

[33] X. Dai, Y. Liang, Y. Zhao, S. Gan, Y. Jia, and Y. Xiang, "Sensitivity Enhancement of a Surface Plasmon Resonance with Tin Selenide ( SnSe ) Allotropes," Sensors, vol. 19, no. 1, p. 173, 2019. 